\title{
Parameter Identification on Lumped Parameters of the Hydraulic Engine Mount Model
}

\author{
Qian $\mathrm{Li}^{1}$, Weibo Liu ${ }^{1}$, Ying Sun ${ }^{1}$, Pei Zhi ${ }^{1}$, Gang He ${ }^{1}$ \\ ${ }^{1}$ Hohai university in Changzhou, China
}

\begin{abstract}
Hydraulic Engine Mounts (HEM) are important vibration isolation components with compound structure in the vehicle powertrain mounting system. They have the characteristic that large damping and high dynamic stiffness in the high frequency region, and small damping and low dynamic stiffness in the low frequency region, which can meet the requirements of the vehicle powertrain mounting system better. The method to identify the lumped parameters of the HEM is not only the necessary work for the analysis and calculation in dynamic performance and can also provide the theory for the performance optimization and structure optimization of product in the future. The parameter identification method based on coupled fluid-structure interaction (FSI) and finite element analysis (FEA) was established in this study to identify the equivalent piston area of the rubber spring, the volume stiffness of the upper chamber, as well as the inertia coefficient and damping coefficient of the liquid through the inertia track. The simulated dynamic characteristic curves of the HEM with the parameters identified are in accordance with the measured dynamic characteristic curves well.
\end{abstract}

\section{Introduction}

HEMs are vibration isolation parts with upper and bottom chambers in rubber mounts; the hydraulic liquid can flow with the pressure change in the upper chamber and bottom chamber. They can provide dual damping mode passive vibration isolators to control high-amplitude, low-frequency road-induced vibrations and lowamplitude, high-frequency engine-induced vibrations.

The general model to analyse the dynamic characteristic of HEMs with inertia track and decoupler is lumped parameter model, some of the parameters can be measured from the product design drawing, but the others can be acquired only through the parameter identification. The empirical formula method uses formulas to calculate the parameters by the relevant theory and experience, the form is relatively simple and the errors of the identification results are large. The relative parameters of lumped parameter model are calculated through the testing for mechanical properties of HEM various components, the result is more accurate, but the testing method need we design and make the frock which be used to test the various suspended components in HEM. So the test process is relatively complex. With the development of computer technology, FEA has been widely used and provided a convenient way to parameter identification. The finite element method can identify the lumped parameter of HEM in design stage. Consequently, we can predict the dynamic characteristics of HEM, and the design can be modify conveniently to satisfy the characteristic requirements of the HEM [1].
Fig. 1 shows the schematic of a HEM studied in this study. The parameter identification method based on coupled FSI and FEA was established to identify the equivalent piston area of the rubber spring, the volume stiffness of the upper chamber, as well as the inertia coefficient and damping coefficient of the liquid through the inertia track. The simulated dynamic characteristic curves of the HEM with the parameters identified are in accordance with the measured dynamic characteristic curves well.

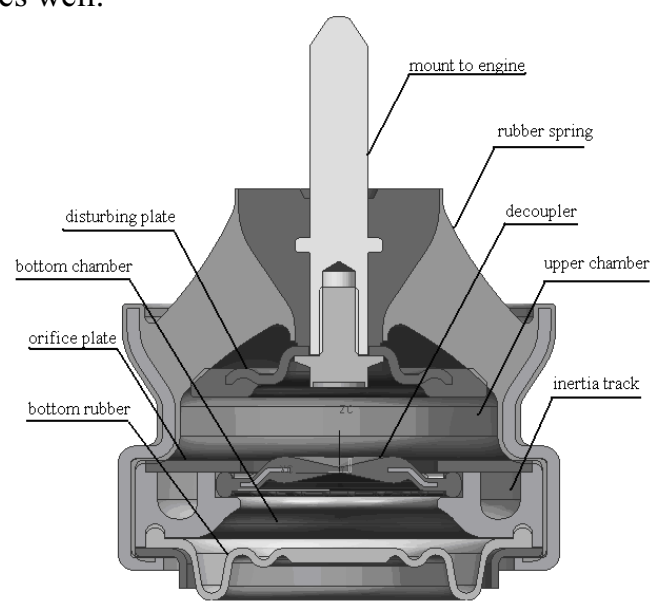

Figure 1. The schematic of a HEM.

\section{The lumped parameter of the HEM}


The principal physical effects are taken to be those associated with the primary rubber including dynamic stiffness and damping, bulge stiffness, piston area and with the inertia track including fluid inertia and damping [2]. The stiffness of the secondary rubber is small enough that it can be ignored for reducing the requisite state dimension by one. The fluid is assumed incompressible for the bulge compliance of the primary rubber is much greater and the fluid inertia and damping in the upper chamber are ignored for inertia and damping in the track is much greater. Considering the HEM in Fig. 1 at large amplitude drive, the LP model for this HEM is shown in Fig.2.

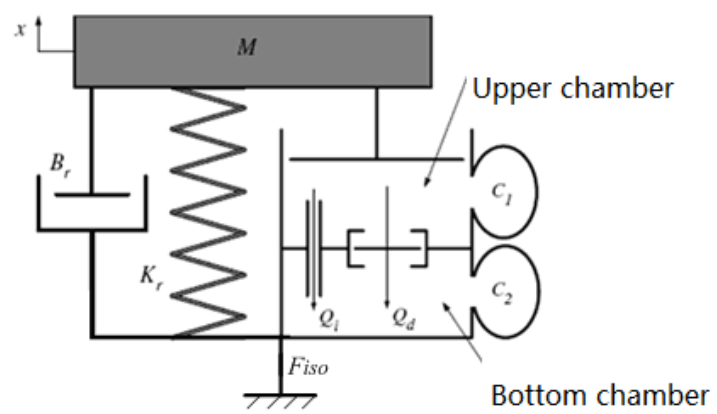

Figure 2. Model of the HEM.

The rubber spring is simplified as one degree of freedom (DOF), and its dynamic stiffness and damping property are described by $K_{\mathrm{r}}$ and $B_{\mathrm{r}}$, respectively. The rubber spring also functions as a piston with an effective area $A_{\mathrm{p}}$. Finally, the rubber spring adds volumetric compliance to the model, represented by $C_{1}$. The reciprocal of the $C_{1}$ is defined as the volumetric stiffness of the upper chamber and is represented by $K_{\mathrm{b}}$. Since the fluid in the chambers is almost incompressible, the volumetric stiffness only depends on the configuration and the Shore hardness of the rubber spring. The fluid in the inertia track is assigned lumped parameters $I_{\mathrm{t}}, B_{\mathrm{t}}$, which represents the track inertia, and track damping, respectively. The lumped inertia parameter, $I_{\mathrm{t}}$, is defined as [1]:

$$
I_{t}=m_{t} / a_{t}^{2}=\rho l_{t} / a_{t}
$$

Where $\rho$ is the density of the fluid in chambers, $m_{t}$ is the fluid mass in the inertia track, $l_{t}$ and $a_{t}$ are length and cross-sectional area of the inertia track, respectively.

The state equation of the model is:

$$
\left[\begin{array}{c}
\dot{x}_{r} \\
\dot{V}_{1} \\
\dot{Q}_{i} \\
\dot{Q}_{d}
\end{array}\right]=\left[\begin{array}{cccc}
0 & 0 & 0 & 0 \\
0 & 0 & -1 & -1 \\
0 & \frac{K_{1}}{I_{i}} & \frac{-B_{i}}{I_{i}} & 0 \\
0 & \frac{K_{1}}{I_{d}} & 0 & \frac{-B_{d}}{I_{d}}
\end{array}\right]\left[\begin{array}{l}
x_{r} \\
V_{1} \\
Q_{i} \\
Q_{d}
\end{array}\right]+\left[\begin{array}{l}
1 \\
A_{p} \\
0 \\
0
\end{array}\right] \dot{x}
$$

The output equation of the model is:

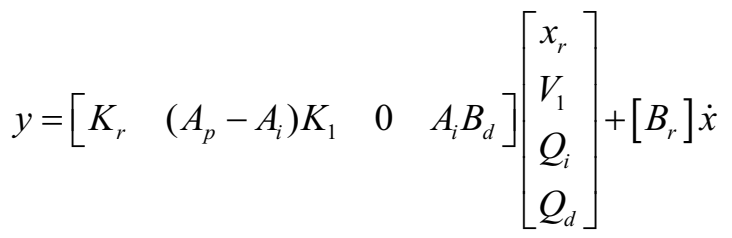

\section{Parameter identification of the HEM model}

\subsection{Identification of the dynamic stiffness and damping of the main rubber spring}

Captions should be typed in 9-point Times. They should be centred above the tables and flush left beneath the figures.

Dynamic stiffness of main rubber spring $K_{\mathrm{r}}$ can be represented by its static stiffness $k_{\mathrm{s}}$

$$
K_{r}=f \cdot k_{s}
$$

Where $f$ is a correction factor which is generally in the range from 1.2 to $1.6 . K_{\mathrm{s}}$ is the static stiffness which can be obtained by experiment or finite element analysis method.

The static stiffness of the main rubber spring can be calculated using FEA method. Fig. 3 is the FEA model of the main rubber to calculate its static stiffness. Fig. 4 is the displacement contour of the spring rubber and Fig. 5 is the displacements of the main rubber spring at different loads. The static stiffness of the main rubber spring can be calculated as the slope ratio of the Fig.5, which equals $151.02 \mathrm{~N} / \mathrm{mm}$. The correction factor $f$ is selected as 1.4 , so the dynamic stiffness of the main rubber spring is $211.43 \mathrm{~N} / \mathrm{mm}$.

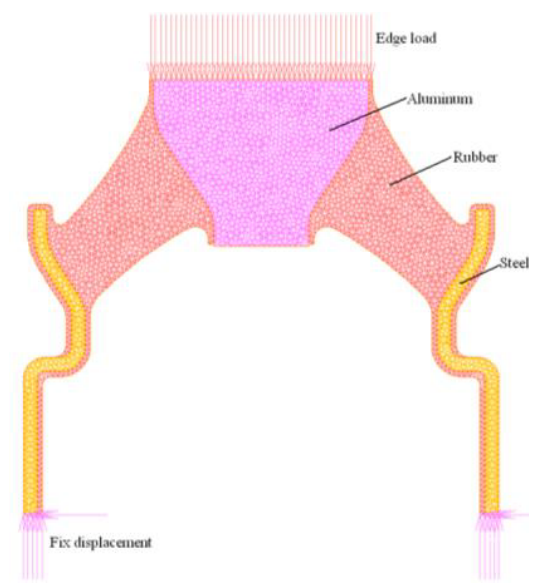

Figure 3. Finite mesh of the spring rubber.

The damping coefficent of the main rubber spring can be calculated using Eq.5.

$$
B_{r}=2 \xi_{r} \sqrt{M K_{r}}
$$

Where $M$ is the mass of the main rubber spring and $\xi_{r}$ is the damping ratio of the rubber material. The mass 
of the main rubber sping is $0.25 \mathrm{~kg}$ and the damping ratio of the rubber material is 0.069 in this study. So the damping coefficent of the main rubber spring can be calculated as $3.17 \mathrm{~N} . \mathrm{s} / \mathrm{mm}$.

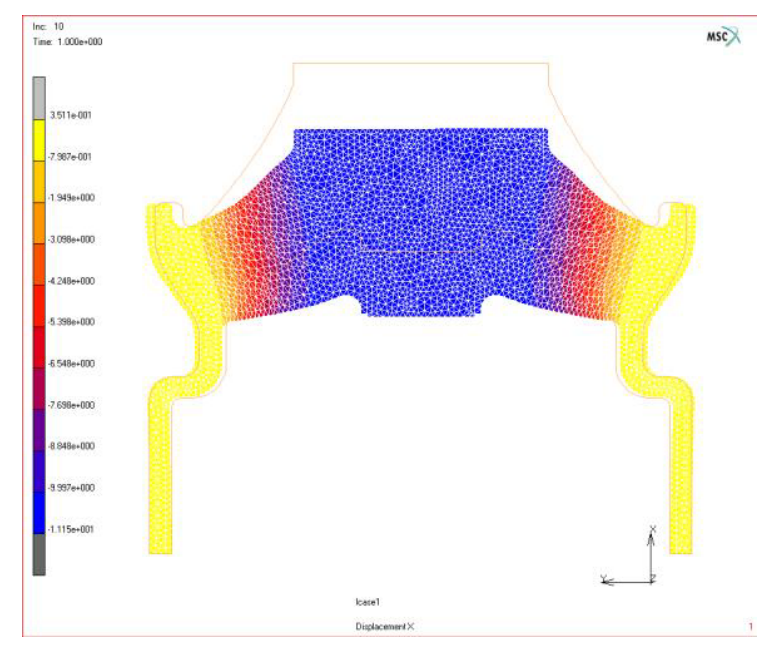

Figure 4. Displacement contour of the spring rubber.

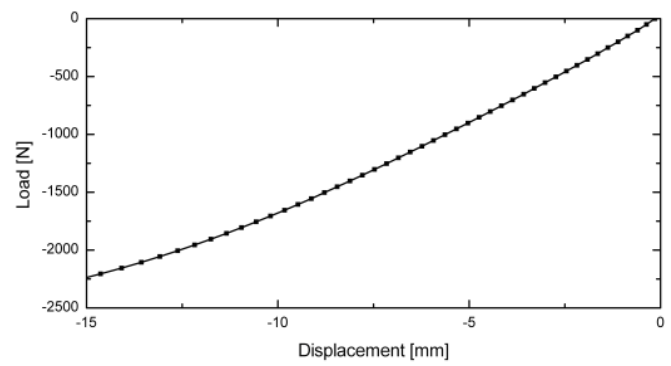

Figure 5. Compression force versus displacement curves of the rubber spring.

\subsection{Identification of the equivalent piston area of the main rubber spring}

The equivalent piston area of the main rubber spring was defined as when the displacement for the upper end of the rubber spring is $x$, the volume of liquid discharged by the main rubber spring equals the volume of liquid discharged by a piston with area $A_{\mathrm{P}}$.

$$
A_{p} x=Q
$$

Deformation of the main rubber apring is generated by the initial load of the engine weight. The initial load can affect the equivalent piston area of rubber spring. The test data and the calculation results show that: when the displacement of the main rubber spring is more than $5 \mathrm{~mm}$ under the action of preload, the equivalent piston area of rubber spring are basically unchanged. Normally, the rubber spring displacement is more than $5 \mathrm{~mm}$, so the equivalent piston area is a constant, which are regardless of the amplitude and frequency of the load. In majority of studies, it usually deal with as constant, the magnitude can be calculated by the method of finite element as follows.

Fig. 6 is the FEA model and boundary conditions for calculating the equivalent piston area of rubber spring.
The equivalent piston area of rubber spring is calculated using Eq. 6 as shown in Fig.7.

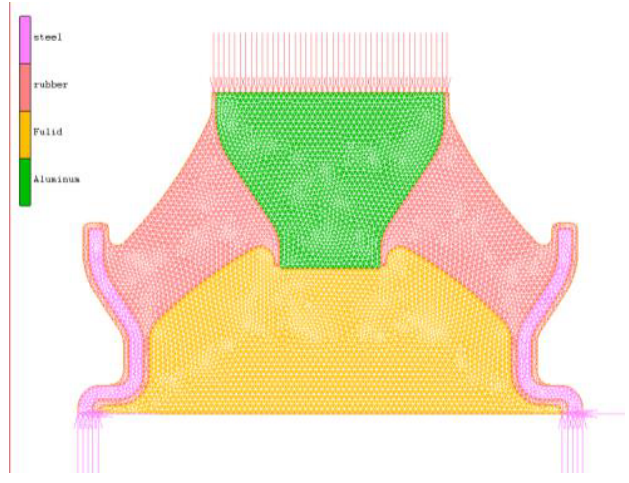

Figure 6. The FEM model.

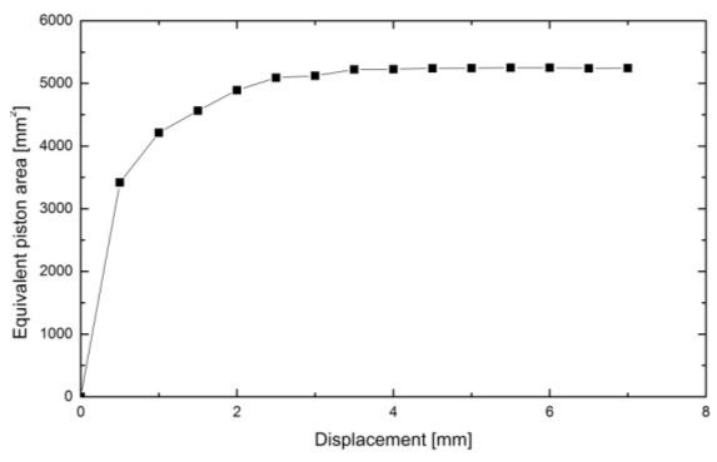

Figure 7. The equivalent piston area of rubber spring at different displacement.

It can be seen from Fig. 7 that the volume-stiffness of upper liquid chamber is basically unchanged when the rubber spring displacement is more than $3 \mathrm{~mm}$, and the equivalent piston area of rubber spring can be obtained as $5.227 \times 10^{3} \mathrm{~mm}^{2}$.

\subsection{Identification of the volume stiffness of upper chamber}

The volume-stiffness of upper chamber is the ratio of the change of pressure vers the change of volume. The mesh model to calculate the volume-stiffness of upper chamber is same to the mesh model to calculate the equivalent piston area of rubber spring, just different in boundary conditions. The finite element model to calculate the volume-stiffness of upper chamber is shown Fig.8.

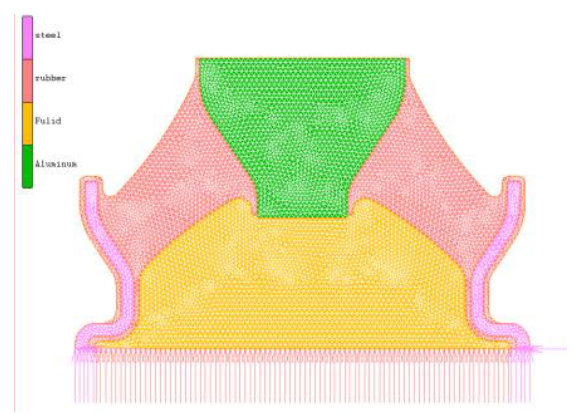

Figure 8. The FEM model. 
Pressure versus volume variation in the upper chamber is shown Fig.9. The volume-stiffness of upper chamber is calculated as $2.423 \times 10^{9} \mathrm{~N} / \mathrm{m}^{5}$.

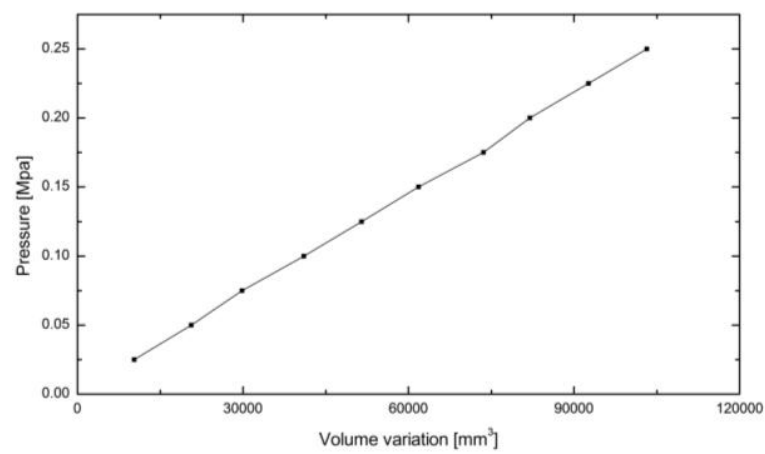

Figure 9. Pressure versus volume variation in the upper chamber.

\subsection{Identification of the inertia coefficient and damping coefficient}

The momentum equation of the liquid flows through the inertia channel is [3],

$$
\Delta P=P_{1}-P_{2}=I_{i} \dot{Q}_{i}+B_{i} Q_{i}
$$

Transform it into the frequency domain,

$$
\frac{\Delta P_{s}}{Q_{s}}=-j \omega I_{i}+B_{i}
$$

So we can obtain the inertia coefficient and damping coefficient of the liquid flow through the inertia track by the amplitude-frequency characteristics between the pressure difference of the two ends of the inertia track and the liquid flow rate of the liquid flow through the inertia track.

Fig. 10 is the finite element model of liquid in the inertia channel established by the software Gambit. The entrance port of inertia track is exerted a pressure load which magnitude is sinusoidal variation with the time and the flow changes in exit port is recorded.

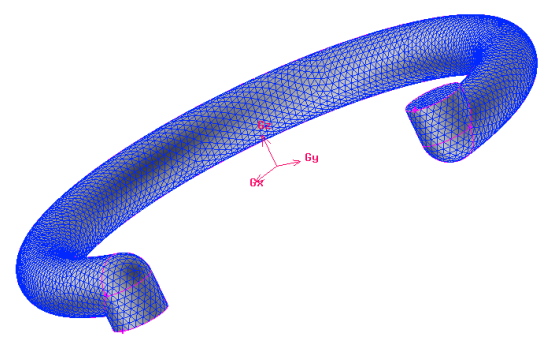

Figure 10. The finite element model for the fluid in the inertia track.
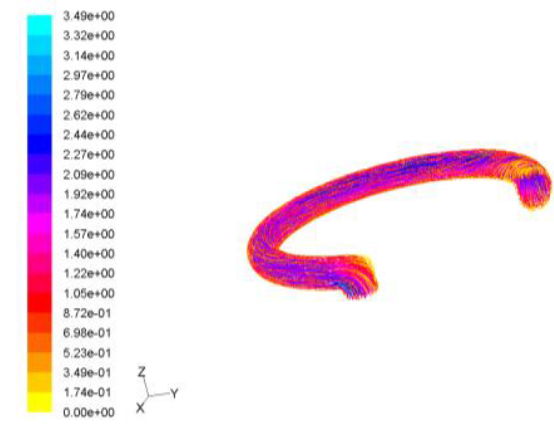

Figure 11. Velocity path lines of the liquid in the inertia track.

Fig. 12 is the inertia and damping in the inertia track at different frequency. The inertia and damping coefficient of liquid flowing through inertia track are calculated using Eq. 8 as $3.154 \times 10^{6} \mathrm{~kg} / \mathrm{m}^{4}$ and $6.366 \times 10^{7} \mathrm{~N} . \mathrm{s} / \mathrm{m}^{5}$.

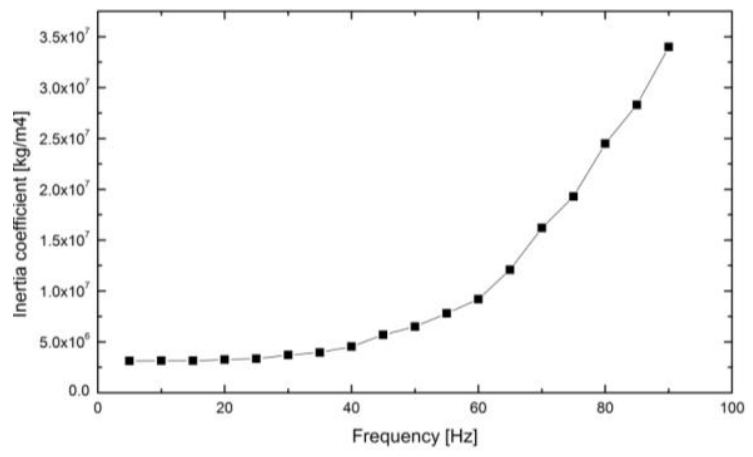

(a) Inertia coefficient

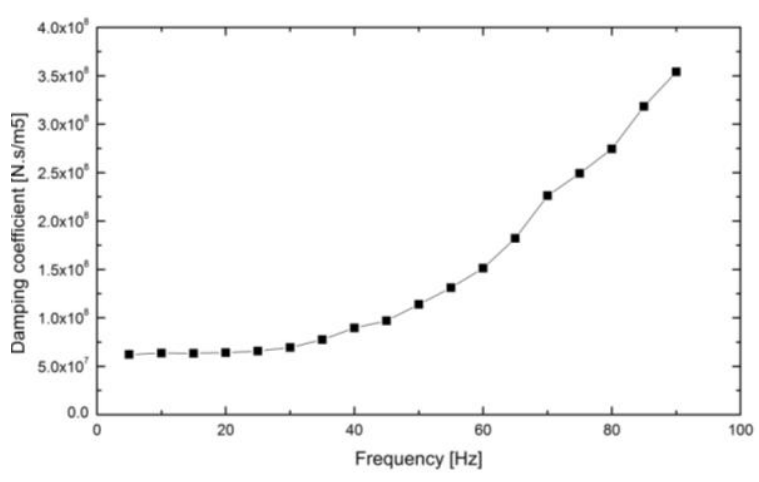

(b) Damping coefficient

Figure 12. Inertia and damping in the inertia track at different frequency

\subsection{Identification of the cross sectional area of inertia track}

After the structure of the HEM is setected, the cross sectional area of the inertia track can be calculated using Eq.1. The cross sectional area of the inertia track is 4.546 $\times 105 \mathrm{~m} 2$.

\section{Results}

The dynamic stiffness and damping of the HEM can be obtained using the parameters identified in this study. 
The simualted and the experiment dynamic characteristics of the HEM is shown in Fig. 13.

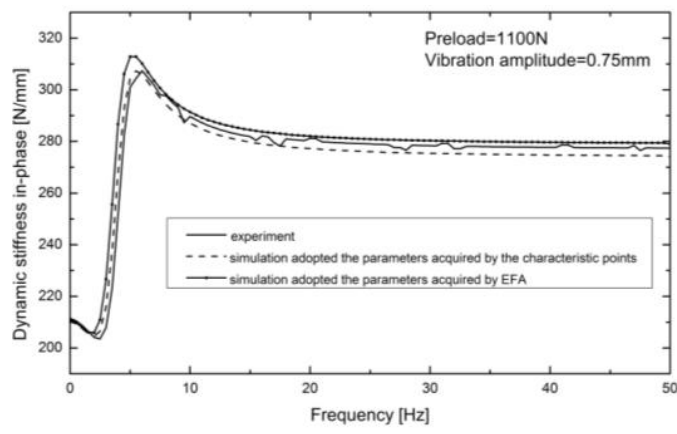

(a) Dynamic stiffness in-phase

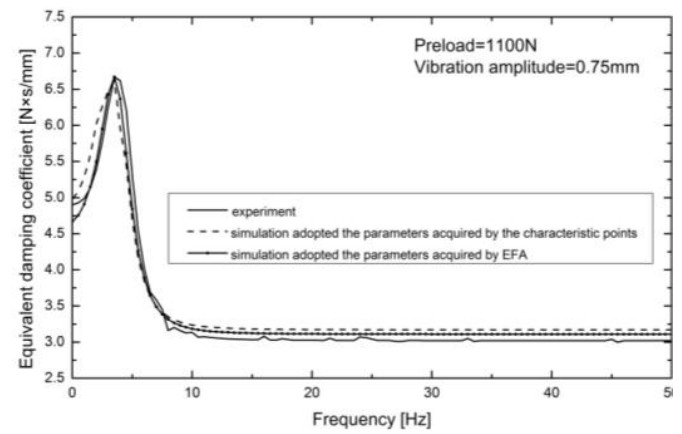

(b) Equivalent damping coefficient

Figure 13. The experiment and simulation dynamic characters of the HEM

\section{Conclusions}

The parameter identification method of the lumped parameters of the HEM based on FSI and FEA is established in this study. The finite element method can identify the lumped parameters of the HEM in design stage. The simulated dynamic characteristic curves of the HEM with the parameters identified are in accordance with the measured dynamic characteristic curves well. Consequently, we can analyse the dynamic characteristics of HEM in the design stage efficiency and reduce the cost of manufacture and test.

\section{Acknowledgments}

This work was financially supported by the Natural Science Foundation of Jiangsu Province of China (Grant No. BK20130237).

\section{References}

1. J. Christopherson, G. Nakhaie Jazar, J SOUND VIB, 290, 4 (2006)

2. R. Singh, G. Kim, P.V. Ravindra, J SOUND VIB, 158, 3 (1992)

3. G. Kim, R. Singh, J DYN SYST-T ASME, 115, 482 (1993) 